\title{
Phase response curve analysis of a morphologically realistic globus pallidus neuron model reveals a distal dendritic mechanism for synchronization

\author{
Nathan W Schultheiss*, Jeremy R Edgerton and Dieter Jaeger
}

Address: Department of Biology, Emory University, Atlanta, GA, USA

Email: Nathan W Schultheiss* - nschult@emory.edu

* Corresponding author

from Sixteenth Annual Computational Neuroscience Meeting: CNS*2007

Toronto, Canada. 7-12 July 2007

Published: 6 July 2007

BMC Neuroscience 2007, 8(Suppl 2):S22 doi: I0.I I86/I47I-2202-8-S2-S22

(c) 2007 Schultheiss et al; licensee BioMed Central Ltd.

\section{Background}

Phase-locked bursting and oscillations in low frequency bands between the subthalamic nucleus (STN) and the globus pallidus (GP) are key features of the pathophysiology of Parkinson's disease (PD). These dynamics may reflect susceptibility of the basal ganglia (BG) to entrainment with cortical oscillations or could also be a consequence of enhanced reciprocal STN-GP coupling under conditions of dopamine depletion.

A.

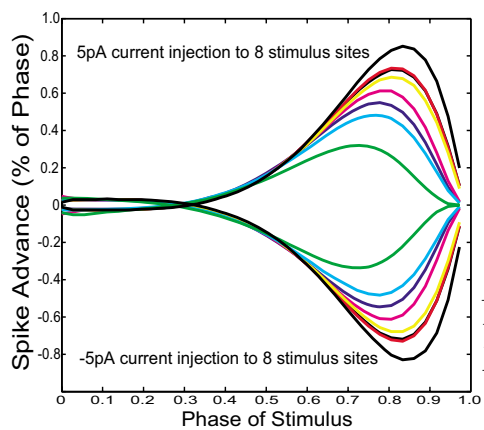

B.

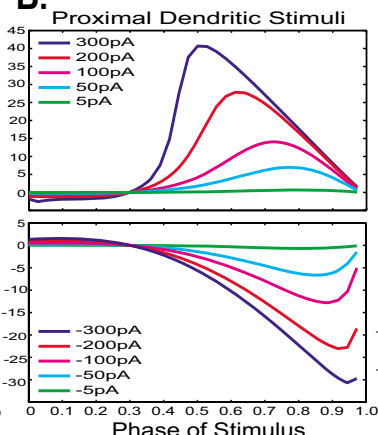

Phase response analysis is an efficient method of characterizing the tendency of single neurons to entrain to periodic input, and to predict the tendency of connected networks to synchronize. A phase response curve (PRC) describes the dependency of shifts in spike timing that result from weak inputs on the timing of inputs within the ongoing inter-spike interval (ISI). If, independent of stimulus phase, a depolarizing input causes an advance of the next spike, the PRC will be composed purely of positive values (a Type I PRC). A Type II PRC contains both positive and negative regions, indicating that a depolarizing

C.

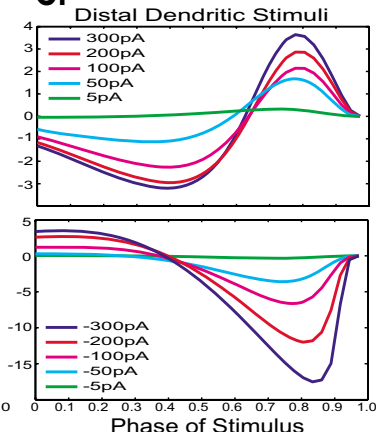

D.

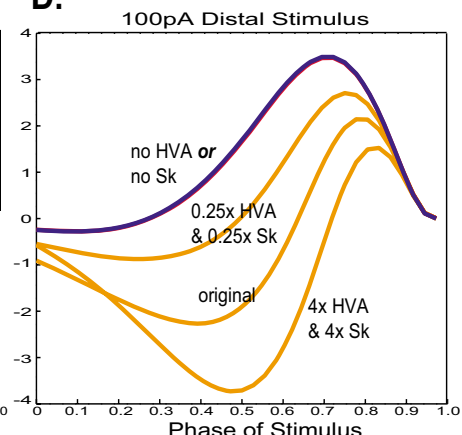

Figure I

Phase response curves. A. PRCs for weak stimuli. B. PRCs for proximal stimuli of different amplitudes. C. PRCs for distal stimuli of different amplitudes. D. PRCs for distal stimuli when HVA and/or SK have been varied. 
input can cause either an advance or delay of the next spike depending on when within the ISI it occurs. Type II PRCs favor synchronization in connected neuronal populations.

\section{Methods and results}

To investigate the phase response properties of GP neurons, we applied simulated current injections and synaptic inputs to a morphologically realistic 585 compartment GP neuron model containing 9 voltage-gated conductances. Stimuli were delivered to one of seven dendritic locations or the soma to determine whether the input site affects significantly the shape of resultant PRCs. When inputs were small (+/-5 pA current injections or equivalent synaptic strengths) PRCs from all eight locations were Type I, and excitatory and inhibitory PRCs were symmetric across zero. (Fig. 1A.) More distal stimulus locations yielded PRCs with increasingly attenuated and left-shifted peaks.

For distal dendritic sites, larger excitatory stimuli resulted in Type II PRCs. (Fig. 1C.) To uncover the mechanism of this stimulus-amplitude-dependent transition between Type I and Type II PRCs, we analyzed differences in distal dendritic currents between control and stimulated conditions. We found that larger stimuli caused increased outward $\mathrm{K}^{+}$flow through the $\mathrm{Ca}^{++}$activated SK channel, and hypothesized that large stimuli delivered to small distal compartments (with high input resistance) activate the high-voltage $\mathrm{Ca}^{++}$channel (HVA) which in turn activates SK. When we locally up- or down-regulated HVA and SK in tandem, the degree of Type II character was correspondingly increased or decreased, and the complete removal of either HVA or SK from the dendrite yielded identical Type I PRCs. (Fig. 1D.)

\section{Conclusion}

Our findings confirm previous work demonstrating PRC attenuation and left-shifting when weak stimuli are applied at increasing distance from the soma. In addition, by using realistic synaptic input, and analyzing evoked active conductances in spatially distinct regions of a realistic model, we characterize a mechanism in distal dendrites for Type II phase response dynamics. As network synchronization is observed in PD but not normal conditions, our findings suggest the hypothesis that GP neurons in PD may receive enhanced distal dendritic excitation and/or show an upregulation of SK conductance.

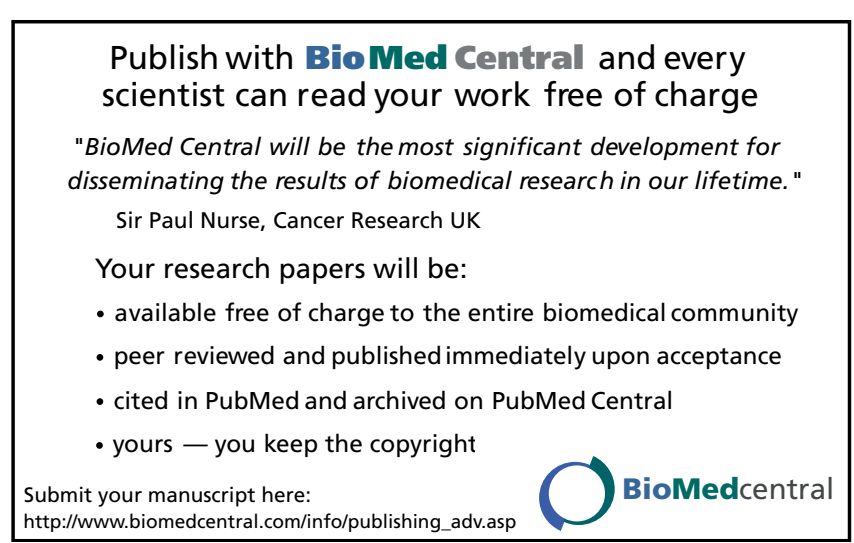

Page 2 of 2

(page number not for citation purposes) 